\title{
RESPOSTA DINÂMICA SOBRE UMA HASTE EM QUEDA LIVRE CHOCANDO-SE TRANSVER- SALMENTE CONTRA UM APOIO RÍGIDO
}

\author{
AleXANDRE U. HofFMANN ${ }^{1}$, WANG CHONG ${ }^{2}$. \\ 1. Grupo de Mecânica Aplicada, Programa de Pós-graduação em Engenharia, Universidade Federal \\ do Pampa, Avenida Tiaraju, $N^{\circ}$ 810, Cep: 97546-550 - Alegrete, RS, BRASIL \\ E-mails: alexandre.hoffmanneibiruba.ifrs.edu.br \\ 2. Grupo de Mecânica Aplicada, Programa de Pós-graduação em Engenharia, Universidade Federal \\ do Pampa, Avenida Tiaraju, $N^{\circ}$ 810, Cep: 97546-550 - Alegrete, RS, BRASIL \\ E-mails: wangchong@unipampa.edu.br
}

\begin{abstract}
The present study is aimed to mathematically model the physical phenomenon that occurs when a cylindrical metal rod hits in free fall run up against a rigid base exactly in the center of its length, this is considered non-deformable rigid base. A closed analytical solution found is the dynamic response of the phenomenon, despite being in the form of series, only the first two items are significant, and the others are discarded. This rod on its surface must withstand abrasion, so it has hardness greater than the hardness found in its core. The answer found enables us to seek a fundamental solution for the analysis of failures in mechanical components that undergoes this type of request. Thus, we find the critical regions on the stem, where tensions are far greater than the yield stress of the material and could cause surface cracks. It is noteworthy that the solution developed is only valid within the elastic limit of the material and only until the moment when the reversal of movement occurs beam.
\end{abstract}

Keywords_ Closed analytical solution, dynamic response, theory of Euler Bernoulli's beam, shock.

Resumo - O presente estudo, tem por objetivo modelar matematicamente o fenômeno físico que ocorre quando uma haste cilíndrica metálica, em queda livre choca-se contra uma base rígida exatamente no centro de seu comprimento, esta base rígida é considerada indeformável. A solução analítica fechada encontrada é a resposta dinâmica do fenômeno, apesar de estar na forma de séries, apenas os dois primeiros itens são significantes, e os outros podem ser desprezados. Esta haste em sua superfície precisa suportar o desgaste por atrito, sendo assim, ela possui uma dureza maior que a dureza encontrada no seu núcleo. A resposta encontrada nos permite buscar uma solução fundamental para a análise de falhas em componentes mecânicos que sofrem este tipo de solicitação. Assim, encontramos as regiões críticas na haste, onde as tensões são bem maiores que a tensão de escoamento do material, podendo vir a ocasionar trincas superficiais. Vale salientar, que a solução desenvolvida só é válida dentro do limite elástico do material e somente até o instante que ocorre a inversão de movimento da viga.

Palavras-chave—Solução analítica fechada, resposta dinâmica, teoria das vigas de Euler Bernoulli, choque.

\section{Introdução}

Fenômenos de impacto acontecem frequentemente nos processos de produção industrial. Por exemplo, podemos citar o processo de moagem das rochas hospedeiras de minério de ferro, onde esferas ou hastes cilíndricas de aço são abandonadas de certa de altura e transformam sua energia potencial em energia de deformação causando a fratura das rochas minerais, quebrando-as em pedaços menores. Para desenvolver esta atividade, estes componentes mecânicos são submetidos a condições abrasivas severas além de enorme impacto. Buscando melhorar a resistência ao desgaste, a superfície da haste geralmente é tratada termicamente, aumentando-se a dureza da sua superfície. Contudo, com o aumento da dureza poderá ocorrer uma diminuição da tenacidade à fratura da haste, causando quebra da mesma, ocorrendo uma parada não programada no fluxo de produção, onerando economicamente ainda mais o processo de extração do minério de ferro. Para balancear esta relação entre dureza e tenacidade, é fundamental conhecer comportamento dinâmico de uma haste chocando-se com rochas. Este estudo analisou o caso em que uma haste cai de certa altura e choca-se transversalmente contra uma rocha, exatamente na metade de seu comprimento. Esta rocha foi considerada como sendo uma base rígida, indeformável. Baseado na teoria das vigas de Euler Bernoulli, este trabalho obteve uma solução fechada em termos de tempo para a viga durante o choque que ocorre entre ela e a base rígida. Na literatura há soluções sobre uma massa que se choca numa viga (Lee, 1952; Karunes, 1960; Liu, 1988) e também uma viga que se choca com outra viga (Xu, 2001), contudo os autores deste trabalho, não encontraram artigos publicados sobre o caso investigado. 


\section{Materiais e Métodos}

\subsection{Estudo sobre vibrações laterais em vigas}

Para aplicarmos a teoria das vigas de Euler Bernoulli, devemos considerar as seguintes premissas, da teoria elementar das vigas:

- Existe um eixo na viga que não sofre tração ou compressão, e o eixo $x$, será posicionado ao longo deste eixo.

- As seções transversais perpendiculares ao eixo neutro no estado não torcido da viga permanecem planas e perpendiculares ao eixo neutro deformado, o que significa que o cisalhamento pode ser desconsiderado.

- O material é linearmente elástico e as propriedades da viga são homogêneas em todas as direções.

Supondo que a haste caia livremente de uma altura $h$ e choca-se em seu centro com uma base rígida, indeformável, como mostra a figura 1, podemos observar a haste no momento exato do choque, totalmente na horizontal, e em questão de instantes, ela esta fletida.

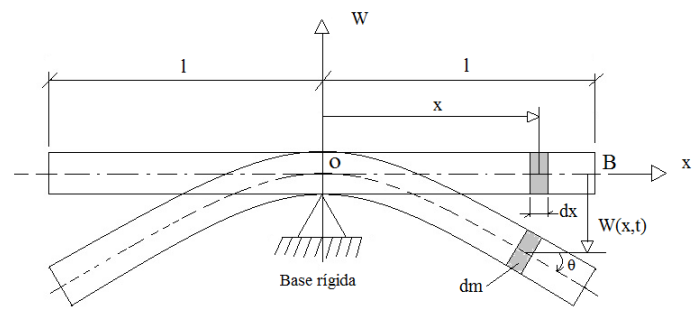

Figura 1. Haste sofrendo o impacto e consequentemente a deflexão

Para uma abordagem mais enfática, iremos dividir a haste exatamente no ponto ' $O$ ' que esta sobre a base rígida, considerando então que a haste possui um vínculo neste ponto, sendo denominada de extremidade fixa e a extremidade ' $B$ ' como sendo a livre, conforme mostrado na figura 2.

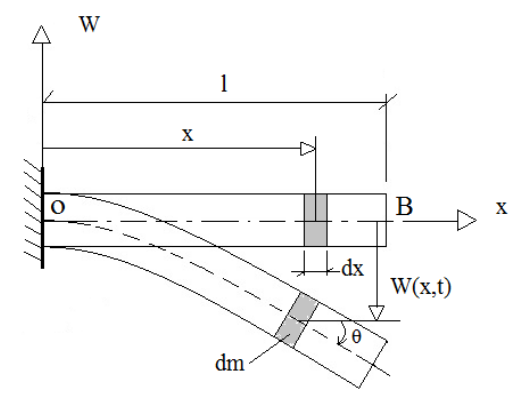

Figura 2. Haste sofrendo o impacto e consequentemente a deflexão

Podemos então analisar um pequeno comprimento da haste, chamado de $d x$, assim, pela figura 3 , podemos observar o diagrama de corpo livre:

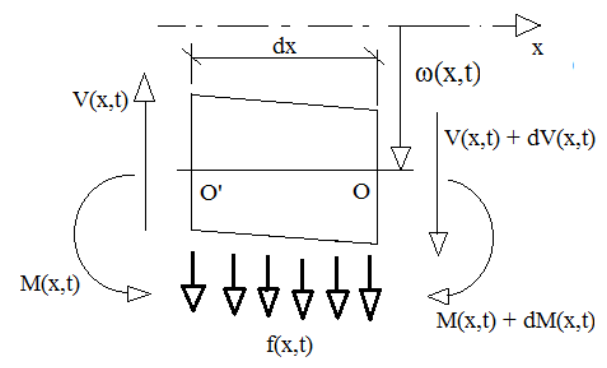

Figura 3. Diagrama de corpo livre

Sendo que $M(x, t)$ é o momento fletor, $V(x, t)$ é a força de cisalhamento e $f(x, t)$ é a força externa por unidade de comprimento da viga, sendo a força de inércia que age sobre o elemento:

$$
\rho A(x) d x \frac{\partial^{2} w}{\partial t^{2}}(x, t)
$$

Onde, $\rho$ é a densidade da massa, e $A(x)$ é a área da seção transversal da viga.

Utilizando a teoria das vigas de Euler Bernoulli, têm-se as relações:

$$
\begin{gathered}
V(x, t)=\frac{\partial M}{\partial x}(x, t) \\
M(x, t)=E I(x) \frac{\partial^{2} w}{\partial x^{2}}(x, t)
\end{gathered}
$$

Denomina-se $E$ como módulo Young do material e $I(x)$ é o momento de inércia da seção transversal da haste.

Através da segunda lei de Newton obtemos a equação de movimento da viga como:

$$
E I \frac{\partial^{4} w}{\partial x^{4}}(x, t)+\rho A \frac{\partial^{2} w}{\partial t^{2}}(x, t)=f(x, t)
$$

Para o caso de vibração livre, $f(x, t)=0$; a equação de movimento torna-se:

$$
c^{2} \frac{\partial^{4} w}{\partial x^{4}}(x, t)+\frac{\partial^{2} w}{\partial t^{2}}(x, t)=0
$$

Onde 'c', é uma constante:

$$
c=\sqrt{\frac{E I}{\rho A}}
$$




\subsection{Solução de vibração livre}

Utilizando o método de separação de variáveis, podemos determinar a solução para vibração livre como:

$$
w(x, t)=W(x) T(t)
$$

Substituindo a equação (7), na equação (4), e rearranjando os termos, obtemos duas equações na forma:

$$
\begin{aligned}
& \frac{d^{4} W(x)}{d x^{4}}-\beta^{4} W(x)=0 \\
& \frac{d^{2} T(t)}{d t^{2}}+\omega^{2} T(t)=0
\end{aligned}
$$

Assim, as frequências naturais da viga são calculadas pela equação (10):

$$
\omega=\beta^{2} \sqrt{\frac{E I}{\rho A}}=(\beta \ell)^{2} \sqrt{\frac{E I}{\rho A \ell^{4}}}
$$

Por consequência a solução para as equações podem ser expressas como sendo:

$$
W(x)=C_{1} \cosh \beta \chi+C_{2} \operatorname{sen} \| \beta \chi+C_{3} \cos \beta \chi+C_{4} \operatorname{sen} \beta \lambda
$$

$\mathrm{C}_{1}, \mathrm{C}_{2}, \mathrm{C}_{3}, \mathrm{C}_{4}$ são constantes diferentes e podem ser determinadas por condições de contorno. Segundo a teoria clássica de viga em balanço (Rao, 2008), a equação característica de autovalores:

$$
\cosh \beta l * \cos \beta l+1=0
$$

Esta equação é satisfeita por um número de $\beta_{n} \ell,(n=1,2,3, \ldots)$, assim:

$W_{n}(x)=C_{n}\left[-\operatorname{sen} \beta_{n} x+\operatorname{senh} \beta_{n} x+\alpha_{n}\left(\cos \beta_{n} x-\cosh \beta_{n} x\right)\right]$

Onde $C_{n}=C_{2}$ e,

$\alpha_{n}=-\frac{C_{1}}{C_{2}}=\frac{\operatorname{senh} \beta_{n} l+\operatorname{sen} \beta_{n} l}{\cosh \beta_{n} l+\cos \beta_{n} l}$

\subsection{Solução de vibração forçada}

Para nosso caso, $f(x, t)=-\rho A g=$ constante $\neq 0, \mathrm{~A}$ solução da vibração forçada de uma viga pode ser determinada pelo método de superposição de modo, para que isto seja possível consideramos que a deflexão da viga seja:

$$
W(x, t)=\sum_{n}^{\infty} W_{n}(x) q_{n}(t)
$$

Onde $q_{n}(t)$ é a coordenada generalizada do enésimo modo de vibração e $W_{n}(x)$ é o enésimo modo normal ou função característica que satisfaz a equação (8). Levando a expressão (15) à equação (40) e através da ortogonalidade de funções $W_{n}(x)$, encontramos a equação:

$$
\omega_{n}^{2} q_{n}(t)+\ddot{q}_{n}=Q_{n}
$$

onde

$$
b_{n}=\int_{0}^{l} W_{n}^{2} d x
$$

$\mathrm{e}$

$$
Q_{n}=-\frac{g}{b_{n}} \int_{0}^{l} W_{n}(x) d x
$$

A solução geral da equação (16) é:

$$
q_{n}(t)=A_{n} \cos \omega_{n} t+B_{n} \operatorname{sen} \omega_{n} t+\frac{Q_{n}}{\omega_{n}^{2}}
$$

Pelas condições iniciais:

$$
w(x, 0)=0 ; \dot{w}(x, 0)=-V_{0}=\text { const } .
$$

Obtivemos a expressão completa do deslocamento lateral da haste após o choque com a base rígida, como:

$$
\begin{aligned}
& w(x, t)=\sum_{n=1}^{\infty}\left[-\operatorname{sen} \beta_{n} x+\operatorname{senh} \beta_{n} x+\alpha_{n}\left(\cos \beta_{n} x-\cosh \beta_{n} x\right)\right] \\
& \frac{d_{n}}{\omega_{n}}\left[\frac{g}{\omega_{n}}\left(1-\cos \omega_{n} t\right)+V_{0} \operatorname{sen} \omega_{n} t\right]
\end{aligned}
$$

Onde $d_{n}$ é igual:

$$
d_{n}=\frac{\left[-2-\left(\cos \beta_{m} l\right)-\left(\cosh \beta_{m} l\right)-\alpha_{m}\left(\sin \beta_{m} l-\sinh \beta_{m} l\right)\right]}{\left\{\begin{array}{c}
{\left[\frac{1}{4}\left(\sinh 2 \beta_{m} l\right)-\left(\sin 2 B_{m} l\right)\right]+\left[-\cosh \beta_{m} l \times \sin \beta_{m} l+\sinh \beta_{m} l * \cos \beta_{m} l\right]+} \\
+\alpha_{m}\left[\left(\cos 2 \beta_{m} l-\cosh 2 \beta_{m} l\right)\right]++2 \alpha_{m}\left[\left(\sin \beta_{m} l * \sinh \beta_{m} l\right)\right]+ \\
+\alpha_{m}{ }^{2}\left[\frac{\beta_{m}}{2}+\frac{1}{4}\left(\sin 2 \beta_{m} l\right)\right]+\alpha_{m}\left[2\left[\sinh \beta_{m} l * \cos \beta_{m} l\right)+\left(\cosh \beta_{m} l * \sin \beta_{m} l\right)\right]+ \\
+\alpha_{m}{ }^{2}\left[\frac{\beta_{m}}{2}+\frac{1}{4}\left(\sinh 2 \beta_{m} l\right)\right]
\end{array}\right\}}
$$




\section{Resultados}

Para realizar esta etapa do trabalho, utilizamos o software Matlab, possibilitando de uma maneira mais rápida a obtenção dos resultados demonstrados abaixo.

\subsection{Dados}

Os dados utilizados foram:

Meio comprimento da Haste: $l=2.21 \mathrm{~m}$;

Raio da haste: $R=0,0508 \mathrm{~m}$;

Módulo de elasticidade: $E=180 \mathrm{GPa}$;

Densidade da haste: $\rho=7850 \mathrm{~kg} / \mathrm{m}^{3}$;

Gravidade: $g=9,81 \mathrm{~m} / \mathrm{s}$;

Altura de queda: $h=6,096 \mathrm{~m}$;

Velocidade no inicio do impacto: $V_{0}=$ $10,9363 \mathrm{~m} / \mathrm{s}$;

\subsection{Obtenção das curvas de distribuição de mo- mento fletor}

Segundo a equação (3), o momento é calculado realizando a segunda derivada de $w(x, t)$ em relação a $x$, sendo assim, temos as figuras abaixo ilustrando os momentos fletores $M_{n}(x, t)$ para $(\mathrm{n}=1,2, \ldots, 10)$ nos instantes $t=0,1 \mathrm{~s}$ mostrado na figura 5, e para $t=0,5 \mathrm{~s}$ é mostrado na figura 6 .

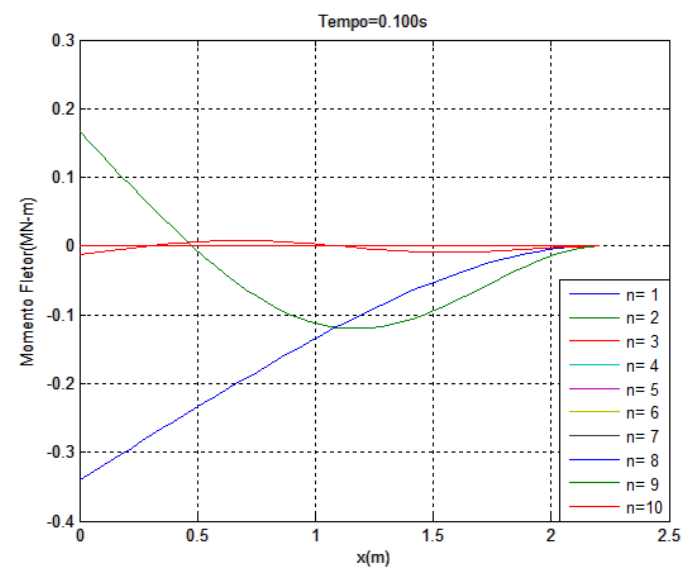

Figura 5. Momento fletor no tempo de 0,1 segundos.

Na figura 6, temos o momento fletor no tempo de 0,5 segundos.

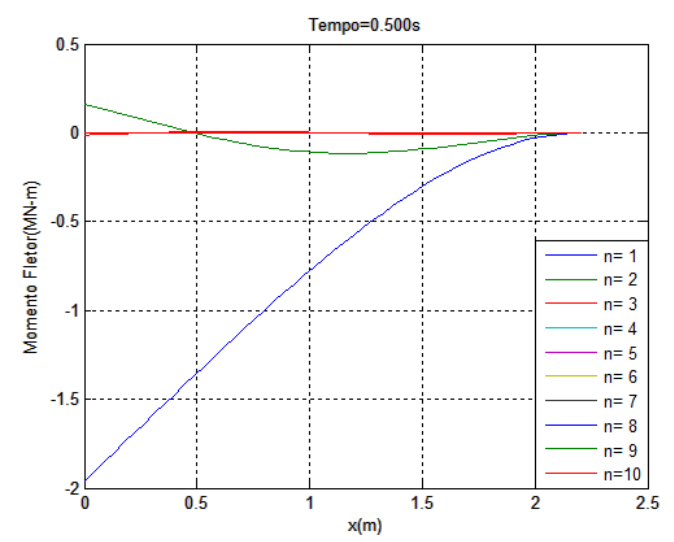

Figura 6. Momento fletor no tempo de 0,5 segundos.

Observando as figuras ficou claro que apenas para os itens $\mathrm{n}=1$ e 2 , os momentos fletores possuem valores significantes. Para $n>2$, os momentos são quase zero, isto nos permite trancar a série da equação (20), quando $n=2$ sem ocorrer maior erro. A figura 7 mostra a variação dos coeficientes $d_{n}$, que decrescem e se aproxima de zero rapidamente quando aumenta o $n$. Isto evidencia que os itens $w_{n}(x, t)$ poderão ser desprezáveis quando $\mathrm{n}>2$.

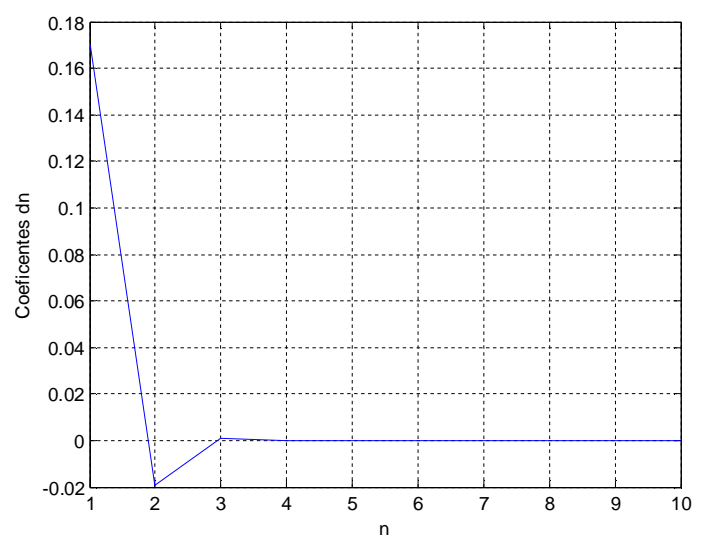

Figura 7 Variação do $d_{n}$ em relação $n$

\subsection{Obtenção dos gráficos de tensões}

A tensão máxima de tração que ocorre na superfície superior da haste é dada por:

$$
\sigma(x, t)=\frac{M(x, t) R}{I}=E I \frac{\partial^{2} w(x, t)}{\partial x^{2}} * \frac{R}{I}
$$

E força cortante é dada por:

$$
V(x, t)=\frac{\partial M(x, t)}{\partial x}
$$

A figura 8 apresenta a distribuição da tensão máxima na superfície superior da barra, variando o tempo de 0 até 0,03 segundos. Através da figura 8, 
percebemos que a tensão máxima que ocorre na superfície da barra não ocorre na posição de contato com o apoio $(\mathrm{x}=0)$, mas aproximadamente a $0,9 \mathrm{~m}$ do apoio. Quando aumenta o tempo, o pico de tensão máxima desloca-se para o apoio.

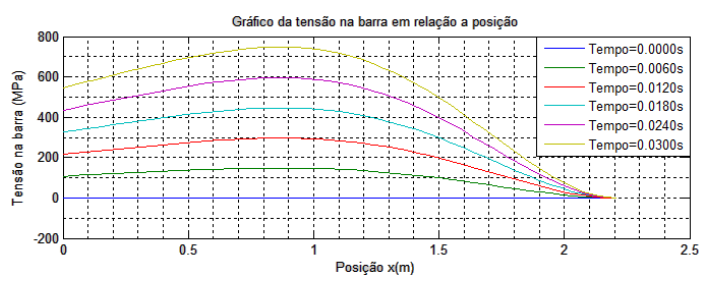

Figura 8. Tensão máxima na haste no intervalo de 0 a $0,03 \mathrm{~s}$.

A figura 9, nos mostra a força cisalhante na haste, podemos observar que é nula na posição da seção transversal onde o momento fletor é máximo.

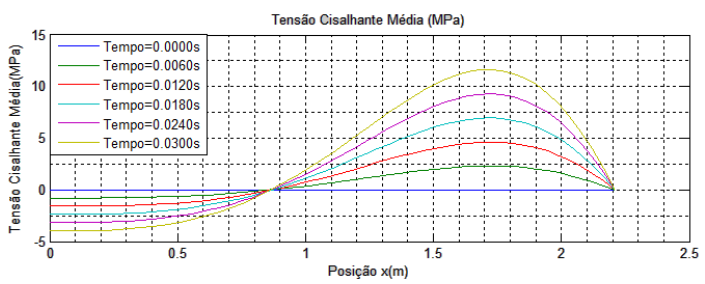

Figura 9. Força cortante na haste no intervalo de 0 a $0,03 \mathrm{~s}$.

Já na figura 10 , foi demonstrado o gráfico da tensão normal na superfície da haste, agora no intervalo de tempo entre 0,3 a 0,4 segundos.

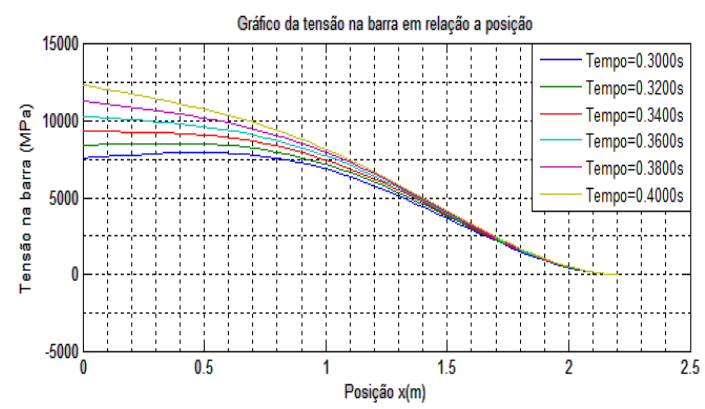

Figura 10. Tensão máxima na superfície superior da haste, no intervalo de 0,3 a $0,4 \mathrm{~s}$.

Podemos observar na figura (10), que a tensão máxima ocorre no apoio quando o tempo está em torno de 0,36 segundos, no qual, a tensão é aproximadamente de $11500 \mathrm{MPa}$. É evidente que antes de atingir esta tensão ira ocorrer o colapso da haste.

\subsection{Obtenção das curvas de deflexão}

Na figura 11, temos seis curvas de deflexão plotadas em um intervalo de tempo que varia de 0 até 0,5 segundos.

Podemos observar neste gráfico que conforme aumenta o tempo, a deflexão vai aumentando.

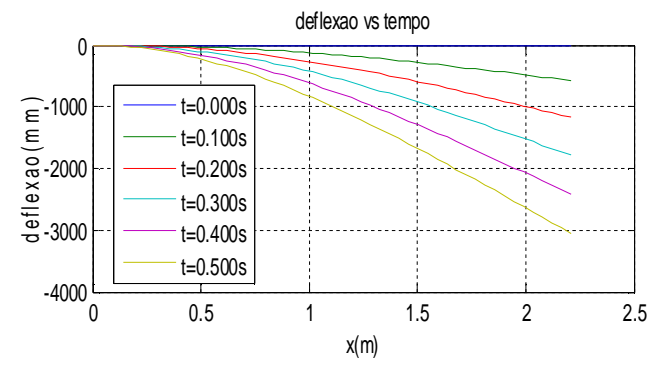

Figura 11. Curvas de deflexões no intervalo de 0 a $0,5 \mathrm{~s}$.

Para que possamos analisar o momento exato quando ocorre o retorno elástico da viga, utilizamos um intervalo de tempo mais refinado, que esta sendo mostrado na figura 13 .

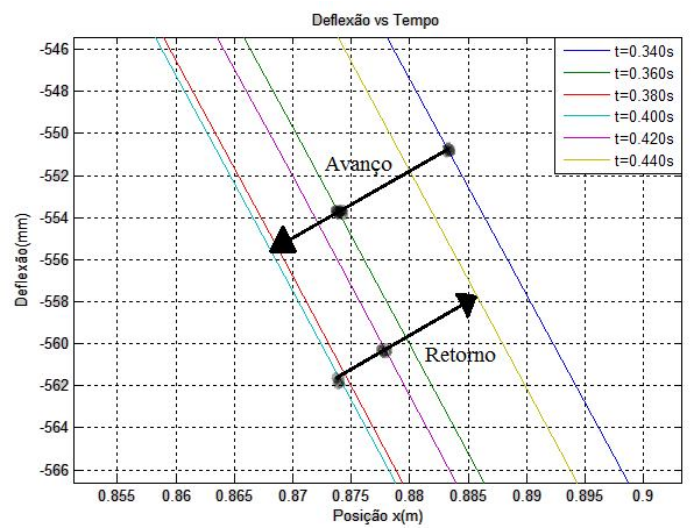

Figura 12. Deflexão na barra no intervalo de 0,34 a 0,44 segundos.

Nela podemos observar que a linha azul representa o tempo de 0,34 segundos, em seguida temos a linha de cor verde com o tempo de 0,36 segundos. Avançando ainda mais temos a linha em vermelho com o tempo de 0,38 segundos, em seguida esta a linha turquesa clara, com o tempo de 0,4 segundos onde ocorre a inversão de sentido e a haste tende a voltar para a sua posição de repouso.

\section{Contribuição deste artigo}

A solução dinâmica encontrada é de suma importância para o estudo da relação existente entre a dureza superficial da haste com a sua tenacidade à fratura. Esta etapa desenvolvida neste trabalho será utilizada para o estudo de propagação de trincas na superfície da haste, bem como, para encontrar uma equação matemática que descreva o melhor perfil de 
transição entre a dureza superficial da haste, com sua tenacidade a fratura do material do núcleo da haste.

\section{Conclusão}

A solução obtida é analítica fechada. Apesar de estar na forma de série, apenas os primeiros dois itens são significantes e os restos poderão ser desprezáveis. A solução poderá servir como uma solução fundamental para análise de falhas na haste de queda livre e chocando seu centro com um stop rígido.

\section{Agradecimentos}

Agradecemos a CAPES pelo financiamento deste trabalho

\section{Referências Bibliográficas}

Filho, E. L. M. (1992). Manual de Redação e Estilo, Maltese.

Karunes, B.; Onat, E.T.; (1960). On the effect of shear on plastic deformation of beams under transverse impact loading. J. Appl. Mech. Vol.27, 107. DOI: 10.1115/1.3643883

Lee, E.H.; Symonds, P.S.; (1952). Large plastic deformation of beams under transverse impact. J. Appl. Mech. Vol. 19, 308.

Liu, J.H.; Jones, N.; (1988). Dynamic response of a rigid clamped beam struck by a mass at any point on the span. Int. J. Solid Struct. Vol. 24, 251. DOI: 10.1016/0020-7683(88)90032-7

Rao, S. S.: (2008). Vibrações Mecânicas. Pearson Prentice Hall, São Paulo - SP.

Xu, T.X., Yang, J.L. Reid, S.R. (2001), Deformable body impact: dynamic plastic behaviour of a moving free \pm free beam striking the tip of a cantilever beam, International Journal of Solids and Structures, No. 38, pp. 261-287.

Boyce, W. E.; Diprima, R. C; (2011). Equações Diferenciais Elementares e Problemas de Valor de Contorno. LTC, Rio de Janeiro - RJ.

Stewart, J. (2011). Cálculo. Vol. 2. Cengage Learning. São Paulo - SP.

Anton, H.; Bivens, I.; Stephen, D. (2007). Cálculo. Vol. 2. Bookman, Porto Alegre - RS.

Balachandra, B.; Magrab, E. B. (2011). Vibrações Mecânicas. Cengage Learning. São Paulo - SP. 